\title{
GSP10
}

\section{Deep Resistivity Images as a Factor in Making Geosteering Decisions}

W Szczawinski* (Baker Hughes Incorporated), S. Noaman (Baker Hughes Incorporated) \& D. Zurcher (Baker Hughes Incorporated)

\section{SUMMARY}

Electrical images produced by azimuthal deep resistivity tools are usually discarded as a source of valuable information due to their lack of recognizable and qualifiable features giving geological information. This paper presents ways to use them in order to support decision making process. 
Electrical image produced by azimuthal deep resistivity tools are usually discarded as a source of valuable information due to their lack of recognizable and qualifiable features giving geological information. For many people involved in geosteering they are purely indicating the direction of closest conductive bed - whether it is from high or low side. And most of the time actually it's true. However there are certain situations when this particular image gives some indication about changing lithological situation. This could be useful especially in case of unexpected events such as sub-seismic faults or dramatic changes in lithology. Additional benefit of using those features is the fact that deep resistivity tool is usually placed very close to the bit giving some advantage over density or gamma images.

Deep resistivity image is not giving information in a traditional way such as density or gamma images. It doesn't draw sinusoidal shapes most of geosteering specialists are used to. It is related to the physics that lays beyond it and also the amount of the formation that is taken into account. The depth of investigation of imaging tools is counted in single inches and it has a good justification what we want to see is the contrast given by different lithologies appearing on contact surfaces intersected by borehole. The bigger volume of rock taken into account the lesser resolution of such method simply just due to statistics. In case of deep resistivity, as the tool is "looking" far into the formation in order to forewarn about incoming changes the volume of rock is quite big so the image is not particularly impressive. However certain changes in the environment are producing certain features that could be recognized and used in favor of taking geosteering decisions.

In this paper we will present theoretical models as well as practical examples where this approach could be used in order to make complex tasks simpler and provide geosteering specialists with powerful tool to le them take their decisions faster. 